\title{
Circulación y reestructuración de la referencia en un diálogo adulto-niño ${ }^{1}$
}

\section{Christian Hudelot}

La presente contribución ${ }^{2}$ tiene por objeto reflexionar sobre lo que he denominado "circulación de la referencia en los diálogos adulto-niño". Hablar de la referencia es interesarse por la manera como el mensaje se refiere a otra cosa distinta de sí mismo, ya sea real o virtual, lingüística o extralingüística3. Nos interesa señalar que la noción de referencia no se inscribe en una perspectiva en la que se trata la lengua como un sistema homogéneo de oposiciones y de combinaciones reguladas, sino que se refiere más al modo de funcionamiento de los elementos lingüísticos en el circuito de la comunicación. De acuerdo con Frédéric François (1984), nos proponemos denominar "lenguaje" a este proceso4. En este artículo tengo solamente la pretensión de avanzar en algunas propuestas de análisis inspiradas esencialmente en la relectura de diálogos entre una madre y su hija de cinco años y un mes. La primera preocupación es señalar la relación entre la lengua y el lenguaje. Para este cometido, me valdré de un triple hilo conductor: lo realizado con el lenguaje en la comunicación, lo lingüístico y lo dialógico.

Sobre el lenguaje, propongo primeramente los diferentes tipos de referencia. Si bien es evidente que los objetos de la referencia pueden variar de objeto, de proceso, de cualidad, de experiencia (común, privada, genérica, específica e incluso indeterminada - por no hablar de las más frecuentemente citadas)-, el modo de referenciación no procede del mismo género de inventario. Se trata de establecer la relación entre el mensaje y su objeto en el contexto de la interlocución, respecto del cual me parece que se podrían al menos señalar cuatro relaciones: la referencia con relación a un "marco" situacional o contextual, la referencia al objeto presente, la referencia compartida, relativa a un objeto ausente, y la referencia construida por el discurso.

En relación con las herramientas lingüísticas de la referencia, recordaremos que, aparte de los medios gramaticales, como la deixis o la anáfora, existen otros, construidos por combinaciones gramaticolexicales tales como la correferencia, la referencia indirecta y la referencia metalingüística. Por último, como tercer hilo conductor, se tomará en cuenta la interlocución en la circulación y la reestructuración de la referencia.

Se tratará sobre todo de mostrar que la referencia no es independiente de las condiciones propias del diálogo ni del conocimiento compartido por los interlocutores ni de los lugares que ellos ocupen, por

1 Tomado de C. Hudelot (1989, pp. 79-91). Traducido por Rosa D. Morales V., Grupo Lenguaje, Cultura e Identidad, Universidad Distrital Francisco José de Caldas.

2 Este artículo es la versión corregida de una comunicación redactada en la Red Europea Adquisición de las Lenguas: Mesa Redonda de la Baume-Lès-Aix, 30 de noviembre al 4 de diciembre 1987.

3 Utilizamos aquí "referencia" para designar el modo de presentación de "algo diferente del texto" real y físicamente presente, real y no directamente perceptible, bien sea generalidad o ficción (Remacle y François, 1986, pp. 151-166).

$4 \quad$ "Analizar la lengua es analizar, según la terminología de Halliday, los potenciales de significación; analizar el lenguaje es estudiar el sentido actual de los mensajes en función de su relación con la situación extralingüística de lo que acaba de ser dicho o de lo que va a ser dicho, sea en un contexto estrecho o en un contexto más amplio" (François, 1987). 
ejemplo, en un intercambio pregunta-respuesta. Y esto porque la lógica de las interrogaciones no es necesariamente la misma de las aserciones, pues, por ejemplo, si usted me pregunta si tengo hijos, yo puedo perfectamente responderle: "sí, tengo uno".

Ya es una costumbre afirmar que los fenómenos de elaboración del discurso aparecen mejor cuando se presentan dificultades en la comunicación. Es por esto que voy a comenzar presentando el ejemplo de la iniciación de un diálogo en el que se dibuja una reestructuración temático-referencial. El investigador acaba de pedirle a la madre de Jeanne que "haga hablar" a su hija, teniendo como motivo la presentación de una imagen en la que se representan actividades tradicionales de una clase de preescolar, luego hablar sobre el futuro y, finalmente, discutir sobre los juegos o juguetes que la niña prefiere ${ }^{5}$. El diálogo madre-hija comienza después de un breve intercambio sobre el refrigerio que Jeanne acaba de tomar.

Madre: Qué piensas de esta, de esta, de \# esta clase\#

Jeanne: \#yo\# [di]dibujo, dibujo y la torta

Madre: ¿Dónde viste la torta?

Jeanne: Y eso (mostrando el dibujo) qué es

Madre: ¡Ah! Pues no está lista eh, la está preparando

Jeanne: ¡Hum!

Madre: No está terminada todavía

Jeanne: Pues para mí, lo que yo, lo que más me gusta, de la clase es hacer galletas y dibujar

Madre: Ah

Jeanne: Ah, voy a dibujar (risas). Deberías saber que era dibujar. ¡No!
Aunque la intervención de la madre es algo que podemos llamar una intervención implicante, la respuesta que le da su hija es de alguna manera opaca, en el sentido de ser difícilmente interpretable. Podemos incluso preguntarnos si la niña se queda en el campo del sentido o más bien en el mundo presentado por la pregunta de su madre. En efecto, en la medida en que el dibujo no está actualizado, es difícil saber a quién se dirige la expresión: ¿a la imagen efectivamente presentada, a la representación de dibujos fijados en las paredes del salón, a la actividad de los niños de la misma clase o a un tipo de actividad que a Jeanne le gusta realizar tanto en la casa como en el colegio? ${ }^{6}$

Respecto a la "torta", no se hace ninguna referencia especial a ella, a pesar de la presencia del artículo, ya que tampoco aquí se puede establecer claramente la relación con el discurso de la madre. Sin embargo, con la nueva alusión que hace la mamá, la torta comienza a funcionar como objeto presente, visible. Notemos que este modo de ver el objeto continúa siendo verdadero, aun en la pregunta del adulto que interpretamos como contraargumento, dando a entender que no se ve la torta. Por otra parte, en esta segunda interpretación es cuando la niña puede replicar con un argumento ad rem: es decir, mediante la exposición de los hechos: "y eso qué es". La contraargumentación de la madre se expresa, entonces, como forma de reutilizar el mismo contenido modificado, puesto que ya había sido presentado primero bajo su "incoactividad" y luego bajo su sola virtualidad. Los dos últimos enunciados de la niña constituyen una salida con respecto al tipo de diálogo en proceso: al

5 Para una presentación más completa de las condiciones de grabación, enviamos a Hudelot y cols. (1982) [el signo \# señala encabalgamiento: los interlocutores producen enunciados al mismo tiempo; N. del Trad.].

6 Estamos de acuerdo con François en decir que "Si 'no podemos no comunicar', 'no podemos no referir', es decir enviar a un mundo más allá del discurso. [...] Sobre todo, podemos considerar que cualquier manera de significar es una manera indirecta de referir; independientemente de toda deixis, hablar de mesa, de verdad o de impresión implica de por sí indicar potencialmente un modo de ser diferente" (1986, pp. 21-59). 
tiempo que se quedan en el mismo universo temático, se envían a mundos diferentes como el de la imagen presente y, de manera correlativa, a modos de ser diferentes (práctica, genérica) de la actividad propia del dibujo.

\section{Modos de referencia}

Analicé el ejemplo anterior para presentar la diversidad de los modos de referencia. Así como no existe un solo lenguaje ni un único uso, tampoco existe un único modo de ser de la referencia. Estos diferentes modos no pueden deducirse a priori de los hechos de la lengua; por tanto, resulta algo arriesgado proponer una lista. Sin embargo, distinguiré al menos cuatro aspectos principales.

LA REFERENCIA ENMARCADA: la noción de enmarcamiento envía, por ejemplo, al hecho de que un elemento puede ser determinado por un artículo definido, si señala un elemento perteneciente a un conjunto que está presente en la situación o que fue presentado anteriormente en el contexto. ${ }^{7} \mathrm{La}$ expresión "los clavos" funciona bien como una referencia definida porque reenvía a la fabricación de una biblioteca. Veamos:

Jeanne: Mi PAPÁ hizo su biblioteca y yo le ayudé (risas)

Madre: Ah sí, mira, hablando de [su] biblioteca, sí, entonces ¿qué era lo que tú hacías?

Jeanne: Eh, yo metía los clavos cuando los mojaba en el jabón

Así mismo, podemos decir que el término "la pesca", a la cual se refiere la niña en el siguiente diálogo, ligado al tema de la fiesta de la escuela, no es ni la del pescador con la red ni aquella de Simón Pedro. ${ }^{8}$

7 Acá solo podemos enviar a Annette Karmiloff-Smith (1979).

8 Se refiere al apóstol Pedro [N. del Trad.].
Jeanne: Voy a la pesca. En la pesca hacemos la pesca

Madre: ¿Qué es la pesca?

Jeanne: Hay unas cosas, eh, cuando, cuando pescamos algo, con una cosa, bueno, cuando la agarramos la ganamos (silencio $11 \mathrm{~s}$ ) ¡Mamá!

OBJETO PRESENTE: en la medida en que la madre y la niña discuten frente a una imagen, las referencias al objeto presente son frecuentes. Pero esto no significa que haya un modo único de referenciación. La relación con el objeto presente puede adquirir diferentes aspectos: por ejemplo, la denominación o la identificación contrastiva, como en el siguiente fragmento:

Madre: (Después de 5 segundos de silencio) ¿Y eso qué es? (el padre, presente en el diálogo, señala dos adultos femeninos que figuran en la imagen). Aquí, mira

Jeanne: Esta es una maestra, pero esa es la señora del restaurante escolar

Madre: Ah ¿sí?

\section{REFERENCIA COMPARTIDA CON UN OBJETO AUSEN-} TE: utilizamos este término para significar que los interlocutores re-presentan una referencia común. Es claro que una tal referencia puede ser compartida en diversos grados por todo individuo adulto, por un grupo sociocultural limitado o por un subgrupo particular. Me parece que este último caso se encuentra en el siguiente extracto, en el cual el descriptor tiene dificultades para reconstruir un referente, lo cual no implica mayores problemas a la pareja madre-hija. El diálogo está ligado al montaje de la biblioteca paterna:

Jeanne: Y yo dormía en los CAJONES, me habían 


\begin{abstract}
puesto una almohada en mi lámpara
Madre: Tranquila, no te preocupes, es cierto. Ah, bueno

Jeanne: (Risas)

Madre: ¿Te instaló algo papá? Sí.

Jeanne: Sí, y pues yo leía, eh/ y eh (el padre junta sus manos encima de la cabeza, los dedos pegados en forma de techo)
\end{abstract}

Madre: Papá te había puesto un pequeño techo. Sí, es cierto

REFERENCIA CONSTRUida: se habla de referencia construida cuando el lenguaje (incluidos los medios no verbales, como en la segunda parte del ejemplo anterior) no sirve para representar aquello a lo cual reenvía el discurso, sino más bien para construirlo discursivamente. Jeanne participó con su clase en un picnic en el bosque.

Madre: ¿Comiste en el bosque? ¿\#no tuviste\# frío así con los brazos descubiertos?

Jeanne: \#Ah, no\# no comimos en el bosque

Madre: ¿No?... ¿Dónde comieron, entonces?

Jeanne: Comimoseh (silencio 9 segundos), había eh

Madre: A ver, dime al menos, no estás contando

Jeanne: Había pasto

Madre: Hum

Jeanne: Y comimos sobre el pasto

Madre: ¿En la escuela?

Volveremos más delante sobre los impases relativos o sobre los logros de esta construcción dialógica de la referencia?.

\section{Herramientas de la referencia}

Los medios de los que dispone el locutor para referir pueden reunirse en cinco procedimientos.

LA DEIXIS: reenvía necesariamente a la situación; en caso de que no hacerlo, puede acompañarse de un gesto demostrativo. Incluiremos las relaciones de persona, pero reconoceremos que yo y tú funcionan más que todo como conectores.

ANAFóricos: agruparemos bajo este término el conjunto de los medios gramaticales especializados en la relación de substitución que solo reenvían (salvo eventuales cambios funcionales) a una codificación anterior.

CoRreferencia: se reserva este término para designar las relaciones de substitución que aportan una relación semántica particular (similitud o diferencia) o algún otro modo de ser del referente.

REFERENCIA INDIRECTA, HABLAR DE UN OBJETO: podemos hablar de un objeto sin que este sea necesariamente nombrado. Tenemos, por ejemplo, una referencia metonímica implícita cuando hablamos del asiento delantero, del volante y del freno de mano, sin tener que nombrar explícitamente el automóvil ${ }^{10}$. Otro ejemplo sería el papel de los relativos correlativos en la determinación de un objeto o de un participante en un proceso (Hudelot, 1980).

Jeanne: Eh, eh, iy además se utiliza para ir a gimnasia y lo meten en la clase ahora!

Las pesas, presentadas en el dibujo, son referidas sin necesidad de ser nombradas.

9 Nuestra intención es diferente de la de F. Jacques (1979), pues él intenta estudiar la referenciación en una situación efectiva donde se mezclan factores que el lógico no necesita tomar en cuenta.

10 Tomamos este ejemplo de F. François et al. (1984). 
REFERENCIA METALINGÜÍsTiCA: hablaremos de referencia metalingüística para señalar que se puede reenviar por encadenamiento al discurso del otro según las condiciones de la referencia.

Madre: Ah bueno, ¿y quién te ha dicho qué es una carrera de caballos? Tú sabes lo que es una carrera de caballos

Jeanne: Pues claro, allá estuvimos. ¡Ah!

Madre: Ah bueno

Jeanne: Y ahí reconocí eso / porque, había barreras largas

Notemos aquí que la referencia es doblemente metalingüística. Lo es en el enunciado de la madre, quien pone en duda lo que B. Noëlley R. Grunig llamarían las instancias de validación de la denominación (1985). También es en las palabras de la niña, quien opone su propia experiencia a la duda del adulto: "Allá estuvimos", expresión acompañada del marcador de argumento “ $\mathrm{AAh!”,} \mathrm{que} \mathrm{viene} \mathrm{apoyado} \mathrm{por} \mathrm{una} \mathrm{referen-}$ cia al reconocimiento que precisa esta descripción.

\section{Pros y contras de la circulación de la referencia}

La realidad es sin duda más compleja que las tipologías que hemos esbozado. Dicotomías como la que opone el discurso del objeto presente al discurso del objeto ausente no se sostienen necesariamente ante la comparación, el reconocimiento o la categorización, cada una con un modo diferente y con distintas maneras de reenviar al presente a otra cosa diferente de él mismo. Entonces, es más importante señalar, por un lado, que los modos de referencia pueden acompañarse de codificaciones privilegiadas, y por otro, que esos modos de codificación no circulan necesariamente de forma homogénea entre los interlocutores.

UN EJEMPLO DE SABER COMPARTIDO, INTERPRETADO: comenzaremos por un ejemplo en el cual la refe- rencia concierne principalmente al saber compartido. La madre introdujo el tema de la "amiguita": una de las "amigas" con quien Jeanne fue al picnic en el bosque. Nada indica en el texto que ella correfiera a Carolina. Me interesaré esencialmente en la referencia de "hacer"; para ello, presentaré un contexto lo suficientemente amplio.

$$
\begin{aligned}
& \text { Madre: [...] Hubieras podido llevarle alguito } \\
& \text { Jeanne: } \begin{array}{l}
\text { bueno, la / ¡bah! } \\
\text { Madre: } \\
\text { ¿Un regalito, no? (Jeanne está realmente } \\
\text { indignada) }
\end{array} \\
& \text { Madre: ¿\#Por qué?\# } \\
& \text { Jeanne: Porque \#sus\# } \\
& \text { Madre: \#la\# última vez ella te regaló una cosita que } \\
& \text { era muy linda que había hecho ella misma } \\
& \text { Jeanne: ... ¿Qué? } \\
& \text { Madre: Tu, [su] medalloncito ese, \#en cerámica\# } \\
& \text { Jeanne: \#Sí pero\# ¿sabes lo que yo le regalé?// Una } \\
& \quad \text { cometa } \\
& \text { Madre: ¿Ah sí? \#No sabía\# } \\
& \text { Jeanne: \#Sí\# una cometa } \\
& \text { Madre: Pero ¿quién te llevó la cometa? /el papá de } \\
& \quad \text { Carolina }
\end{aligned}
$$

Jeanne: Sí/ en una caja de cartón

"Hacer" no envía al mismo tipo de actividad, según se trate de la obra de la amiguita o de la obra de Jeanne. Es decir, que hacer un "medallón" o una "cometa" no solamente no son actividades del mismo tipo, sino, sobre todo, que la madre sabe -porque ella conoce las capacidades de su hija, las costumbres de la clase, la generosidad de ciertos padres, etc.- que su hija no fabricó un juguete así de complejo.

No insistiremos más en este punto, sino solamente en el hecho de que las determinaciones del medallón no sirven tanto para identificarlo como para valo- 
rarlo y justificar así mismo la idea de un regalo de vuelta.

REFERENCIACIÓN CONSTRUIDA: la descripción es evidentemente una de las maneras de referir en la cual el discurso funciona de algún modo como una señalación indirecta. Solo transcribiremos un ejemplo:

Madre: Me DIJISTe AHORITA QUe habÍAs VISTO mujeres en las carreras de caballos. No entendí lo que querías decir

Jeanne: Fuimos A Ver EH, los caballos/ estuvimos/ donde ellos entrenan los caballos

Madre: Sí

Jeanne: En una, casa. Y había dos mujeres que se subieron sobre eh un caballo

Madre: Sí

Jeanne: Eh, tenían un caballo cada una

Nos sorprenderemos de la precisión suplementaria "un caballo cada una", que, me parece, es redundante, si aceptamos que en el universo común los jinetes, salvo alguna excepción -en el circo u otra acrobacia- están solos sobre sus monturas ${ }^{11}$.

LA REFERENCIA SE CONSTRUYE EN LA INTERACCIÓN: el acuerdo entre las mentes no se hace automáticamente, pues cada uno puede construir la referencia por su propia cuenta. Vemos, por ejemplo, cómo las "barreras" siguen modos de referir diferentes. Su circulación en el modo como la madre retoma el referente modificado lleva a una reestructuración de la referencia en el discurso de la niña, quien nos describe, aunque con algunos problemas, caballos tal vez "ordenados" o, más prosaicamente, las "barreras" que delimitan la pista usada por los caballos. He aquí el ejemplo: Se acaba de hablar de cómo recordaban las carreras de caballos. Nadie había mencionado las apuestas urbanas ni nada parecido.

$$
\begin{aligned}
& \text { Jeanne: Yo reconocí eso/ porque, había grandes ba- } \\
& \text { rreras } \\
& \text { Madre: Saltaban las barreras } \\
& \text { Jeanne: No, no las saltaban } \\
& \text { Madre: ¿No? } \\
& \text { Jeanne: No porque estaban afuera y ellos estaban } \\
& \text { adentro }
\end{aligned}
$$

En los dos últimos casos, las "barreras" están definidas o por la actividad de los caballos o por la relación espacial de estos últimos con ellas. Pero la referencia puede quedarse bloqueada. En el ejemplo anterior, la interpretación del otro interlocutor había tenido un papel positivo. La especificación de las barreras como objetos que se saltan condujo a la niña a intentar hacer más precisa su descripción. Por el contrario, los excesos de especificación, la mención de "suecos" por oposición a "botas", que es lo que se espera que use un jinete, y la de los "tacones" como una posesión eventual o no, por ejemplo, un lugar -de fijación de las espuelas- cierra el siguiente intercambio.

Jeanne: Y había una señora que tenía suecos para montar

Madre: ¿Suecos de madera? ¿No tenía botas?

Jeanne: Son suecos

Madre: ¡Ah ¿̇sí?! Bueno

Jeanne: Las cosas de los tacones, como así

Madre: $A h$ ¿sí? Ella tenía tacones para subir y pues se entrenó

Jeanne: Hum

11 A menos que en el espíritu de los niños esto signifique que las jinetes no montan cada una un solo caballo una sola vez, como puede suceder en ciertos carruseles. 
Terminaremos con un último ejemplo donde se manifiesta aquello que algunos llamarían una negociación interactiva y que yo prefiero simplemente considerar como el juego de la no variación del significante y la variación de la referencia, por un lado (cfr. escuela primaria) o, por el contrario, mantener un referente común (y sin duda compartido), a pesar de sus modalidades de puesta en palabras en el hecho de que al menos en el discurso de la madre, "ladrona" y "robada" son correferentes.

\section{Allá en esa escuela roban cosas}

Madre: (suspira) Oh oh (6 segundos) bueno, a ver, hablemos de la escuela. Eh, ¿qué piensas, qué vas a hacer en esa escuela? ¿Ah?

Jeanne: Pues No sÉ

Madre: ¿Hablan a veces con Michelle de la escuela?

Jeanne: ah pues mira, allá en esa escuela roban cosas

Madre: Sí, eh, ¿hablaste de eso con Michelle, de lo que vas a hacer y todo? Ya sabes, la escuela es, se va a poner \#serio\# eh

Jeanne: \#¡oye!\# te dije que habían robado algo

Madre: Pues no, no fue ella

Jeanne: Había un osito en la clase

Madre: Pues ella, tal vez ella, cuando ELLA LLEGó a la clase, ella tenía su osito, tal vez en el brazo, lo dejó y luego lo volvió a coger en la noche para salir con su mamá. \#¿Ves?\#

Jeanne: \#Tal vez\# pues no se me había ocurrido

Madre: Pues no hay que hablar demasiado rápido (4 segundos) no quiero que hagas eso ¿eh? (2 segundos)

Podemos subrayar que este extracto presenta dos movimientos ligados al discurso dominante de la madre. Son las preguntas las que dominan al principio. Ellas acompañan el tema de la escuela, presen- tado, según me parece, primero como genérico. La segunda parte está dominada principalmente por una conducta explicativa, con una "escuela" que envía esta vez a un lugar específico en el cual se desarrollan situaciones particulares.

La madre presenta primero la "escuela", con lo que hemos nombrado un apoyo débil (Hudelot, 1989) que no le proporciona a la niña un punto de vista a partir del cual pueda relacionar su propio discurso. Siguiendo en el modo genérico, la escuela es luego presentada como un objeto de discurso (Michèle, se entiende, es el nombre de la profesora) antes de ser retomada en otro discurso. Jeanne, después de una primera negación, continúa con un enunciado, el cual, diremos, está ligado temáticamente, pero no implicado de manera categórica, sino indicando un cambio de mundo. Ya no se trata de la escuela que sucede al preescolar, sino de aquella que ocupa un edificio específico. "En esa escuela (¿referencia compartida?) roban cosas". Podemos preguntarnos por el sentido exacto del demostrativo. Solo diremos que si los "actualizadores" permiten interpretar la relación referencial de los lexicales, estos últimos actúan en consecuencia sobre la interpretación de los gramaticales. Pero acá nos encontramos desarmados frente a la opacidad del término "cosa".

La segunda parte nos permite adivinar que el robo del que habla Jeanne es, bajo la forma que sea, una experiencia compartida, indicada por el deíctico "ella" y, además, según parece, por la correferencia del "oso" y del objeto robado. Desde ese momento se observa que la madre no ignoró el cambio del mundo genérico al particular, en términos de una referencia no apropiada. Es más, Jeanne anuncia este cambio por medio de la fórmula "ah pues mira" (a propósito de la escuela, imagínate que). Si existen distorsiones aquí es porque la madre decide ocupar un lugar diferente en la interacción, utilizando otro juego de lenguaje en el cual la referencia, aquí ex- 
presada como reprimenda, comienza a funcionar de manera diferente (ficción tal vez combinada con la referencia atemporal de la partida).

Podemos ver fácilmente cómo, en un primer movimiento, la referencia del "osito" es al inicio un ejemplo particular de "cosas", especificado luego por "algo" y correferido por la madre como la posesión de un personaje que los interlocutores no especificarán más adelante. Más tarde, regresa el tema del "osito" (cosa robada) a "su" osito (cuya pertenencia es inferida por un comportamiento habitual supuesto) y se hace coexistir a la ladrona y al osito en mundos diferentes, pero no necesariamente extraños.

Habrían podido desarrollarse otros ejemplos. Intentamos mostrar aquí lo que podía ser la circulación de la referencia dentro de un diálogo asimétrico entre adulto y niño, y sería por lo tanto vano sacar conclusiones antes de haber siquiera intentado analizar otros tipos de diálogos, en otras condiciones de interlocución. Esperamos al menos haber mostrado la necesidad de tener en cuenta no solamente la repetición de un referente manifestado en la cohesión o la coherencia de los discursos, sino también la necesidad de considerar, igualmente, la capacidad de los interlocutores para administrar la diferencia y lo inesperado en la heterogeneidad de la connivencia.

\section{Referencias}

Remacle, Nadine y François, Frédéric (1986). Organización y memorización del texto en el caso de tres personas de edad hospitalizadas. CILL 12 (1-2), 151-166.
François, Frédéric et al. (1986). Análisis del funcionamiento discursivo y patología del lenguaje. CILL 12 (1-2), 21-59.

François, Frédéric et al. (1984). Conduites linguistiques chez le jeune enfant. París: PUF.

Hudelot, Christian y cols. (1980). ¿Qué es la complejidad sintáctica? El ejemplo de la relativa. $L a$ linguistique, 16 (2), 5-41.

Hudelot, Christian y cols. (1982). Stratégies discursives et milieux sociaux, études de dialogues adultes-enfants dans deux situations contrastées. Recherche Milieu-sociolinguistique, 2. Univ, París V.

Hudelot, Christian y cols. (1989). Role et place de l'adulte au cours de quelques dialogues adultes enfant (pp. 95-106). L’Interaction. París: Buscila.

Hudelot, Christian y cols. (1989). Circulación y reestructuración de la referencia en un diálogo adulto-niño. CALaP, 6, 79-91.

Jacques, Francis (1979). Dialogiques, recherches logiques sur le dialogue. París: PIF.

Noëlle, Blanche y Grunig, Roland (1985). La Fuite du sens. La construction du sens dans l'interlocution (pp. 56-73). París: Hatier-Crédif.

Karmiloff-Smith, Annette (1979). Functional approach to child language. A study of determiners and reference. Cambridge: CUP. 